\title{
Cardiovascular magnetic resonance imaging: clinical implications in the evaluation of connective tissue diseases
}

This article was published in the following Dove Press journal:

Journal of Inflammation Research

II May 2017

Number of times this article has been viewed

\author{
Sophie Mavrogeni' \\ George \\ Markousis-Mavrogenis' \\ Loukia \\ Koutsogeorgopoulou ${ }^{2}$ \\ Genovefa Kolovou' \\ 'Onassis Cardiac Surgery Center, \\ Athens, Greece; ${ }^{2}$ Department of \\ Pathophysiology, Laikon Hospital, \\ Athens, Greece
}

\begin{abstract}
Cardiovascular magnetic resonance imaging is a recently developed noninvasive, nonradiating, operator-independent technique that has been successfully used for the evaluation of congenital heart disease, valvular and pericardial diseases, iron overload, cardiomyopathies, great and coronary vessel diseases, cardiac inflammation, stress-rest myocardial perfusion, and fibrosis. Rheumatoid arthritis and other spondyloarthropathies, systemic lupus erythematosus, inflammatory myopathies, mixed connective tissue diseases (CTDs), systemic sclerosis, vasculitis, and sarcoidosis are among CTDs with serious cardiovascular involvement; this is due to multiple causative factors such as myopericarditis, micro/macrovascular disease, coronary artery disease, myocardial fibrosis, pulmonary hypertension, and finally heart failure. The complicated pathophysiology and the high cardiovascular morbidity and mortality of CTDs demand a versatile, noninvasive, nonradiative diagnostic tool for early cardiovascular diagnosis, risk stratification, and treatment follow-up. Cardiovascular magnetic resonance imaging can detect early silent cardiovascular lesions, assess disease acuteness, and reliably evaluate the effect of both cardiac and rheumatic medication in the cardiovascular system, due to its capability to perform tissue characterization and its high spatial resolution. However, until now, high cost; lack of interaction between cardiologists, radiologists, and rheumatologists; lack of availability; and lack of experts in the field have limited its wider adoption in the clinical practice.
\end{abstract}

Keywords: connective tissue diseases, cardiovascular magnetic resonance imaging

\section{Introduction}

Cardiology has various noninvasive imaging modalities at its disposal, such as the rest-stress electrocardiogram, rest-stress echocardiography, nuclear imaging, and, more recently, cardiovascular magnetic resonance (CMR) imaging to detect cardiac involvement in connective tissue diseases (CTDs). Exercise electrocardiogram is an important predictive test to select patients either very unlikely or very likely to present cardiac events. However, it is plagued by a low predictive value for intermediaterisk patients. Stress echocardiography, an operator-dependent technique, is based on the induction of a transient worsening in regional function by means of dobutamine pharmacological stress. It has equal diagnostic and prognostic value as radionuclide stress perfusion, but without radiation exposure and with a significantly lower cost. Although being an important imaging modality for patients with suspected coronary artery disease (CAD), stress myocardial perfusion scintigraphy has serious limitations including radiation exposure, imaging artifacts, and low spatial resolution that precludes detection of small myocardial lesions that commonly occur in patients with CTDs. ${ }^{1}$
Correspondence: Sophie Mavrogeni Onassis Cardiac Surgery Center, 50 Esperou Street, 175-6I, P. Faliro, Athens, Greece

Tel/Fax +30 2l 09882797

Email somal3@otenet.gr 
CMR is a recently developed noninvasive, nonradiating, operator-independent technique. Until now it has been successfully used for the evaluation of a number of cardiac diseases including congenital heart diseases, iron overload, valvular and pericardial diseases, cardiomyopathies, and great and coronary vessel diseases. ${ }^{2-13}$ Recently, its diagnostic capabilities have also been extended to the evaluation of cardiac inflammation and to stress-rest myocardial perfusion. ${ }^{14,15} \mathrm{CMR}$ has the important advantage of being able to reveal the pathophysiologic background of cardiovascular lesions in patients with CTDs, even in those with silent disease. However, until now, high cost; lack of interaction between cardiologists, radiologists, and rheumatologists; as well as lack of availability and expertise have limited its wider adoption in clinical practice. ${ }^{16}$

\section{How does it work?}

CMR images are derived from radio-wavelength photons, thus precluding the need for ionizing radiation as in other imaging modalities. These photons are produced by protons (hydrogen nuclei), which are present in abundance in the human body as part of water and lipid molecules. All subatomic particles inherently rotate around their axes. The total angular momentum of a nucleus is called the spin. However, since protons are charged particles, they generate a magnetic field as they rotate around their axes; the vector of this magnetic field is called the magnetic moment, and its direction is perpendicular to the rotational plane of the spin. In nature, the direction of each magnetic moment is random. During a CMR investigation, the patient is subjected to a powerful exogenous magnetic field generated by the main magnetic coil of the scanner. The randomly spinning protons are forced to orient their magnetic moments in a specific direction, facing either parallel or antiparallel to the vector of the exogenous magnetic field, although the majority align themselves parallel to the field vector as this state is preferable (due to lower energy). The protons are then excited by a radiowave frequency electromagnetic radiation pulse. This radiowave frequency pulse disturbs the alignment of the protons, and by providing additional energy, it causes a certain proportion of them to change the direction of their magnetic moments to the antiparallel direction, a higher energy state. After reaching this higher energy state, the protons begin reverting to the lower energy state of parallel direction. As this occurs, the magnetic moments begin to realign to their original, low-energy orientation in the exogenous magnetic field, while the excess energy is re-emitted as radio-wavelength photons. This process is called relaxation, and the relaxation of the net vector of protons is attributable to two distinct but simultaneous processes, referred to as longitudinal (T1) and transverse (T2) relaxation times. The absorbed radiowaves emitted during relaxation are digitized and can be used to provide important information about human tissues. By using smaller magnetic fields generated by three sets of gradient coils (one for each of the $x, y$, and $z$ axes), the main magnetic field can be manipulated so that high-quality images of specific sections of the human body can be generated. This last possibility makes CMR a far more versatile and robust technique than other imaging modalities, which are limited to axial sections of the body.

In MR examinations, a pulse sequence is defined as a combination of sets of radiowave frequency pulses with the application of magnetic fields through the main and gradient magnetic coils, with the goal of imaging specific tissue types. Basic pulse sequences used in CMR are gradient-echo, which can generate a cine loop (Figure 1), T1 images, usually used for anatomic evaluation and for perfusion-fibrosis assessment (Figure 2), and T2 images (Figure 3), commonly used for the evaluation of myocardial edema and assessment of disease acuteness. Late gadolinium-enhanced images are T1 images taken 15 min after intravenous administration of the paramagnetic contrast agent gadolinium and allow the detection of myocardial fibrotic tissue (scar), which appears as a bright area in a background of nulled, black myocardium - "bright is dead" (Figures 4 and 5). However, diffuse myocardial fibrosis could be missed by traditional late gadolinium-enhanced imaging in cases where the entire myocardium is affected, as occurs with systemic sclerosis (SSc). In these cases, T1 mapping and extracellular volume

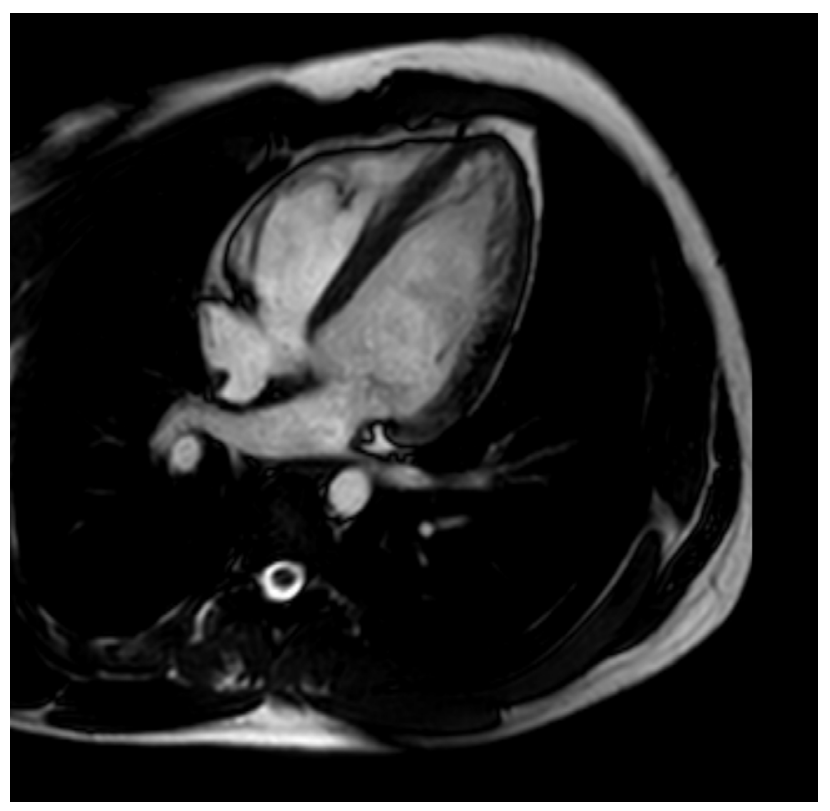

Figure I Four-chamber gradient echo image used for function assessment. 


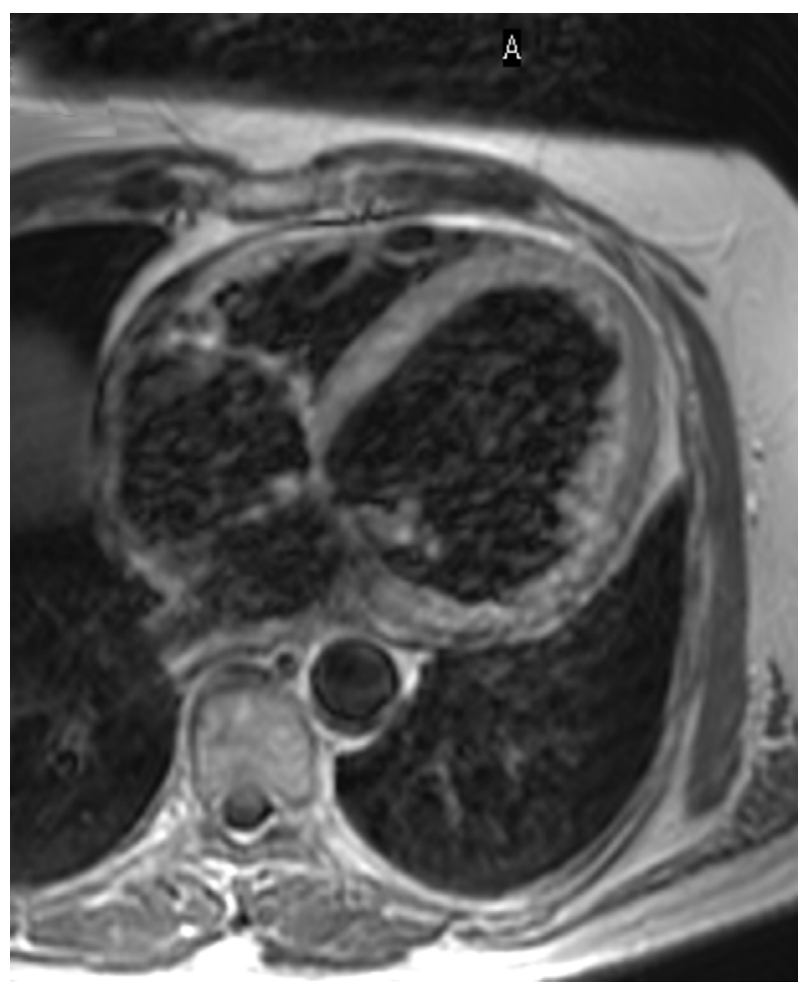

Figure 2 Four-chamber TI imaging used for anatomical assessment.

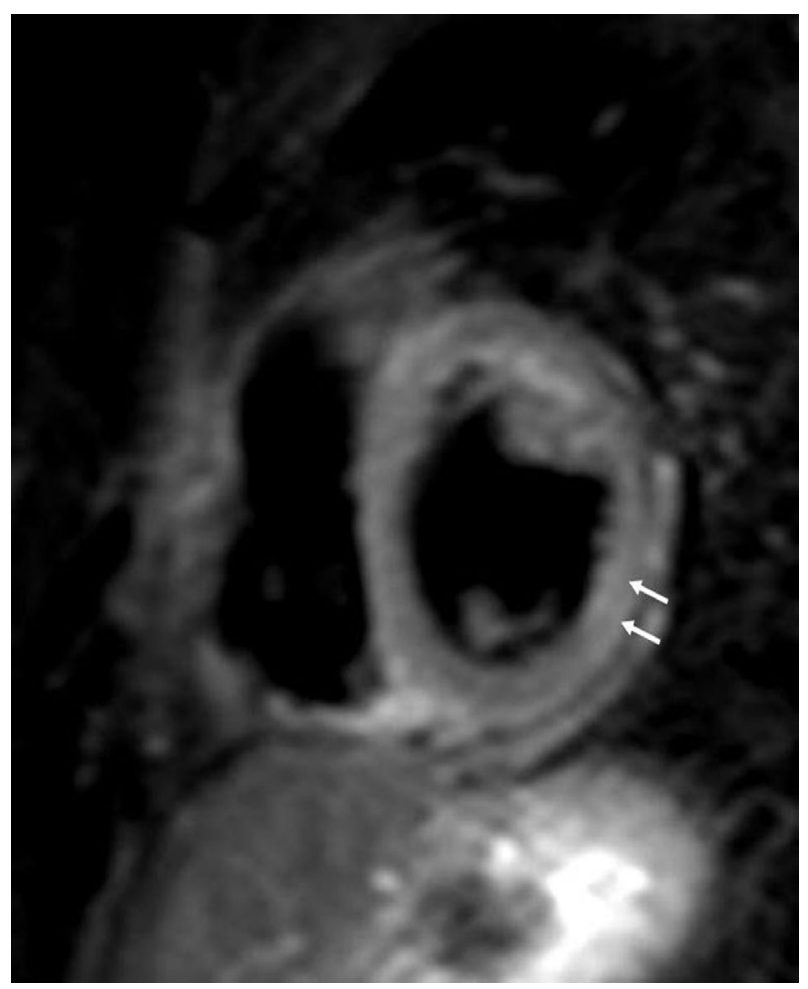

Figure 3 Short-axis T2 imaging from a patient with SLE and myocardial edema. Note: The arrows show areas of myocardial edema in a patient with SLE. Abbreviation: SLE, systemic lupus erythematosus.

quantification can provide a more reliable surrogate estimation of diffuse fibrosis. Finally, noninvasive angiography of vessels can also be performed with the use of a bolus injection of gadolinium (Figure 4). ${ }^{16}$ The use of these

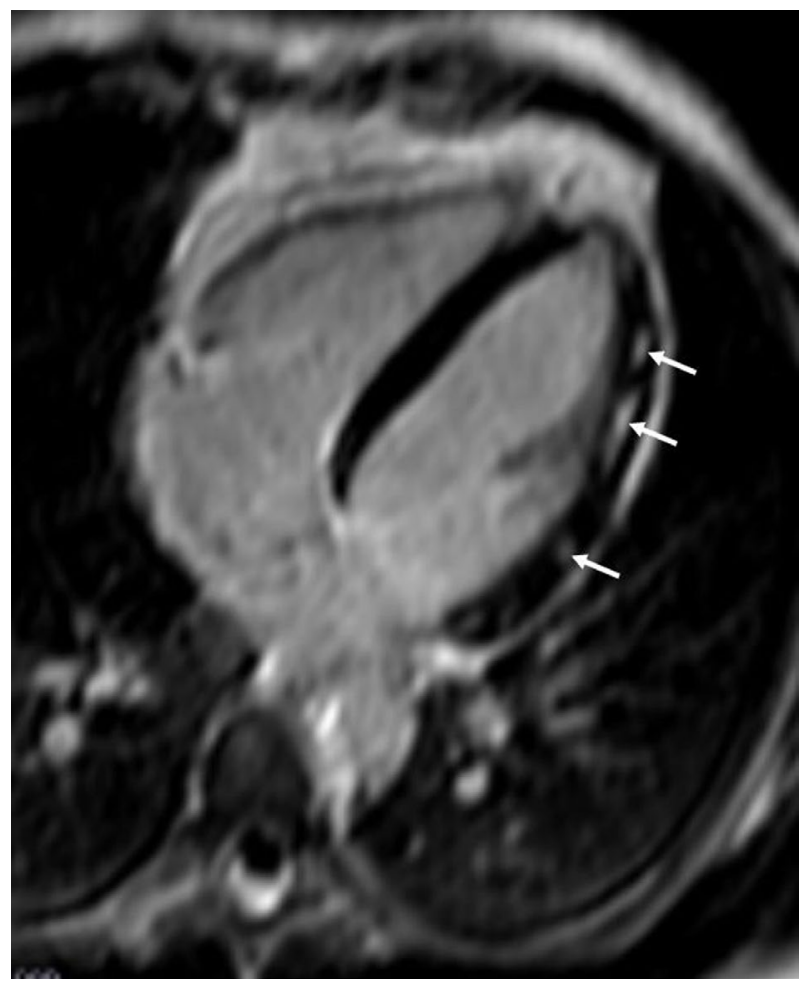

Figure 4 Patchy fibrosis due to myocardial inflammation in a patient with polymyositis.

Note: The arrows show areas of patchy fibrosis in the lateral wall of LV, due to myocardial inflammation in a patient with polymyositis.

Abbreviation: LV, left ventricle.

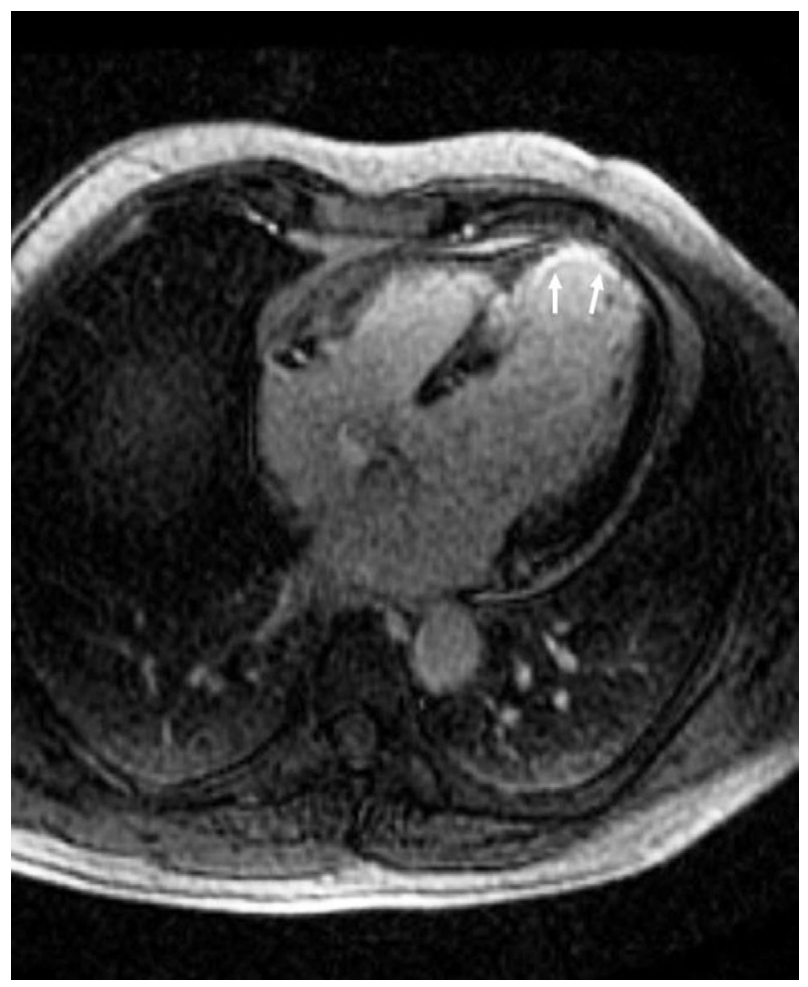

Figure 5 Transmural myocardial infarction in a SLE patient.

Note: The arrows show area of transmural myocardial infarction in a patient with SLE. Abbreviation: SLE, systemic lupus erythematosus. 
sequences allows for reliable assessment of both right and left ventricular volumes and ejection fractions; early detection of myocardial inflammation, which is notably an ability unique to CMR; early detection of perfusion defects due to either macro/microvascular disease; and early assessment of localized or diffuse myocardial fibrosis. ${ }^{16}$

\section{Patients with CTDs presenting with cardiovascular involvement Rheumatoid arthritis and the spondyloarthropathies}

Accelerated atherosclerosis leads to a twofold higher incidence of CAD, stroke, heart failure, and peripheral arterial disease. ${ }^{17,18}$ However, the high incidence of heart failure cannot be explained only by CAD. ${ }^{19,20}$ Other prevalent pathologic entities in rheumatoid arthritis include pericarditis, myocarditis, and vasculitis. ${ }^{17,18}$ Similar problems may occur in the spondyloarthrotopathies. ${ }^{21}$

\section{Systemic lupus erythematosus (SLE)}

Cardiovascular disease is a major cause of morbidity and mortality in SLE and is due to CAD, vasculitis, and myocarditis. $^{22-24}$ The prevalence of CAD is $6 \%-10 \%$, and the risk of developing CAD is 4-8 times higher in SLE compared with controls. Clinically overt myocarditis appears in $3 \%-15 \%$ of SLE, usually in association with pericarditis; however, it is more common at autopsy, suggesting subclinical presentation. ${ }^{22-24}$

\section{Systemic vasculitides}

The typical lesions in vasculitis include inflammation and fibrinoid necrosis of the blood vessel wall. The classification of systemic necrotizing vasculitides depends on the predominant type of vessels affected. ${ }^{25}$ Large-vessel vasculitides includes giant cell and Takayasu arteritis and involves the aorta and its major branches. Medium-vessel vasculitides includes polyarteritis nodosa and Kawasaki disease, while small-vessel vasculitides includes Wegener's granulomatosis, microscopic polyangiitis, Churg-Strauss syndrome, and mixed cryoglobulinemic vasculitis. Based on biopsy findings, inflammation in the myocardium and small vessels may contribute to cardiac ischemia and heart failure. ${ }^{26}$ Vasculitis in SLE, SSc, rheumatoid arthritis, and Sjögren's syndrome affects small-sized vessels, and despite not being clinically predominant, they can be life-threatening. ${ }^{27}$

\section{Inflammatory myopathies}

Heart involvement in polymyositis/dermatomyositis varies between $6 \%$ and $75 \%$ and may be clinically overt at diagnosis, after initiation of treatment, or even during remission; it is usually clinically silent and may lead to fatal arrhythmias or heart failure. ${ }^{28}$

\section{SSc}

$\mathrm{SSc}$ is also called scleroderma and is characterized by involvement of small vessels, resulting in fibrosis of various organs, including the heart. ${ }^{29}$ Cardiac mortality is responsible for $15 \%$ of all scleroderma deaths and is either primary, due to myocardial inflammation-fibrosis, or secondary, due to pulmonary arterial hypertension..$^{30,31}$

\section{Mixed CTDs}

Raynaud's phenomenon of the small vessels of the heart, similar to that observed in the fingers, and development of pulmonary arterial hypertension are included among the most important cardiac lesions in mixed CTDs. ${ }^{32}$

\section{Sarcoidosis}

Cardiac involvement occurs in $20 \%-30 \%$ of sarcoidosis (SRC) patients and conveys a poor prognosis. ${ }^{33}$ Autopsy findings confirmed that granulomas may occur in the myocardium in up to $50 \%$ of fatal SRC and cardiac dysfunction, with sudden death in up to $67 \%$ of cardiac SRC. ${ }^{33}$ Despite characteristic autopsy findings, only $5 \%$ of SRC patients present with clinically overt cardiac disease and only $40 \%-50 \%$ of them are correctly diagnosed during lifetime. ${ }^{21} \mathrm{CMR}$ can detect early cardiac involvement in SRC, including both inflammation and fibrosis as well as perfusion defects. ${ }^{34}$

\section{Clinical implications of CMR in CTDs}

Although considerable improvement has been achieved in both the diagnosis and treatment of CTDs, premature mortality remains a serious, unresolved issue. ${ }^{16}$ Cardiovascular disease in CTDs has been underdiagnosed and undertreated because of a number of contributing factors. The silent and confusing symptoms observed in these patients combined with the lack of accurate diagnostic tools capable of detecting asymptomatic cardiovascular lesions are of major concern in this regard. Additionally, the relapsing nature of CTDs poses great difficulties in the identification of underlying cardiovascular disease, as acute lesions may be superimposed on preexisting areas of fibrosis. ${ }^{16}$

Currently, the most commonly used noninvasive diagnostic tool for investigating cardiac involvement in CTD patients is echocardiography, due to high availability, portability, low cost, lack of radiation, and great expertise among cardiologists. However, this technique cannot distinguish between 
possible etiologies of myocardial dysfunction with accuracy. Furthermore, it is operator dependent, is inherently limited by the requirement for an acoustic window, and cannot perform tissue characterization to detect early silent lesions. ${ }^{35}$ On the other hand, nuclear techniques used in cardiology practice for the assessment of myocardial ischemia-fibrosis have the disadvantages of high cost, radiation exposure, and low spatial resolution, while not allowing the diagnosis of myocardial edema and subepicardial, intramyocardial, or subendocardial fibrotic lesions, which are frequently found in CTD patients. ${ }^{16}$

By means of performing tissue characterization, CMR allows early diagnosis and better understanding of CTD pathophysiology and involvement in cardiovascular disease. Particularly in SSc, where myocardial disease is the result of inflammatory, fibrotic, and microvascular lesions, CMR has a greater sensitivity (75\%) in detection compared to echocardiography $(48 \%){ }^{36}$

Clinical implications of CMR in the risk stratification of CTD patients include

1. Early cardiovascular diagnosis in CTD patients, including inflammation, perfusion defects, and diffuse or localized fibrosis, even if these patients have silent or oligosymptomatic presentation. ${ }^{12,14}$

2. Detection of cardiovascular disease acuteness that cannot be achieved with echocardiography or by nuclear techniques. ${ }^{37,38}$

3. Large-vessel angiography with simultaneous assessment of arterial wall inflammation that can neither be performed with echocardiography nor with nuclear techniques. $^{37}$

4. Tissue characterization that cannot be obtained in an accurate way with echocardiography, nuclear techniques, and cardiac computed tomography. ${ }^{37,38}$ These techniques may suggest the presence of potential lesions, but offer no definitive information.

5. Pharmacologic stress CMR, a noninvasive, nonradiating alternative for stress echocardiography. As not constrained by the limitations of the requirement for an acoustic window and/or the occurrence of breast artifacts, CMR is the technique of choice for coronary artery and cardiac microvascular disease assessment. This is especially the case in females, who constitute the majority of CTD patients and are commonly unable to perform exercise at an adequate level due to arthritis or muscular discomfort. ${ }^{39}$

6. Vigilant long-term cardiovascular monitoring is required in CTD patients due to both the effects of the systemic disease on the cardiovascular system and the cardiovascular burden of medications used for the management of systemic inflammation. CMR can identify high-risk patients and evaluate treatment efficacy and toxicity-related side effects on the heart. ${ }^{40,41}$

7. Cardiac lesions detected by CMR in patients with CTDs can motivate the early commencement of protective cardiac medication according to American College of Cardiology/American Heart Association Guidelines and the modification of rheumatic medication if myocardial inflammation is observed although the systemic disease seems quiescent. ${ }^{42-44}$

\section{Conclusion}

The complicated pathophysiology of CTDs calls for a versatile, noninvasive, nonradiative diagnostic tool suitable for early cardiovascular diagnosis, risk stratification, and treatment follow-up. CMR can detect silent cardiovascular lesions early, assess disease acuteness, and reliably evaluate the effect of both cardiac and rheumatic medication on the cardiovascular system due to its capability to perform tissue characterization and its high spatial resolution. Therefore, its clinical use is strongly recommended in patients with CTDs.

\section{Disclosure}

The authors report no conflicts of interests in this work.

\section{References}

1. Mavrogeni S, Markousis-Mavrogenis G, Kolovou G. The Sphinx's riddle: cardiovascular involvement in autoimmune rheumatic disease. BMC Cardiovasc Disord. 2016;16(1):204.

2. Diethelm L, Dery R, Lipton MJ, et al. Atrial-level shunts: sensitivity and specificity of MR in diagnosis. Radiology. 1987;162:181-186.

3. Mavrogeni SI, Markussis V, Kaklamanis L, et al. A comparison of magnetic resonance imaging and cardiac biopsy in the evaluation of heart iron overload in patients with [beta]-thalassemia major. Eur $J$ Haematol. 2005;75(3):241-247.

4. Wagner S, Auffermann W, Buser P, et al. Diagnostic accuracy and estimation of the severity of valvular regurgitation from the signal void on cine magnetic resonance imaging. Am Heart J. 1989;118:760-767.

5. Masui T, Finck S, Higgins CB. Constrictive pericarditis and restrictive cardiomyopathy: evaluation with MR imaging. Radiology. 1992;182:369-373.

6. Nienaber CA, von Kodolitsch Y, Nicholas V, et al. The diagnosis of thoracic aortic dissection by noninvasive imaging procedures. $N$ Engl J Med. 1993;328:1-9.

7. Kim WY, Danias PG, Stuber M, et al. Coronary magnetic resonance angiography for the detection of coronary stenosis. NEngl J Med. 2001; 345:1863-1869.

8. Manning WJ, Li W, Edelman RR. A preliminary report comparing magnetic resonance coronary angiography with conventional angiography. N Engl J Med. 1993;328:828-832.

9. Post JC, van Rossum AC, Bronzwaer JG, et al. Magnetic resonance angiography of anomalous coronary arteries: a new gold standard for delineating the proximal course? Circulation. 1995;92:3163-3171.

10. Mavrogeni S, Papadopoulos G, Douskou M, et al. Magnetic resonance angiography is equivalent to $\mathrm{x}$-ray coronary angiography for the evaluation of the coronary arteries in Kawasaki disease. J Am Coll Cardiol. 2004;43(4):649-652. 
11. Mavrogeni S, Papadopoulos G, Douskou M, et al. Magnetic resonance angiography, function and viability evaluation in patients with Kawasaki disease. J Cardiovasc Magn Reson. 2006;8(3):493-498.

12. Mavrogeni S, Manoussakis MN, Karagiorga TC, et al. Detection of coronary artery lesions and myocardial necrosis by magnetic resonance in systemic necrotizing vasculitides. Arthritis Rheum. 2009;61(8): $1121-1129$.

13. Kwong RY, Chan AK, Brown KA, et al. Impact of unrecognized myocardial scar detected by Cardiovascular magnetic resonance imaging imaging on event-free survival in patients presenting with signs or symptoms of coronary artery disease. Circulation. 2006;113(23):2733-2743.

14. Mavrogeni S, Manoussakis MN. Myocarditis and subclavian stenosis in Takayasu arteritis. Int J Cardiol. 2011;148(2):223-224.

15. Schwitter J, Wacker CM, van Rossum AC, et al. MR-IMPACT: comparison of perfusion-Cardiovascular magnetic resonance imaging with single-photon emission computed tomography for the detection of coronary artery disease in a multicentre, multivendor, randomized trial. Eur Heart J. 2008;29(4):480-489.

16. Mavrogeni SI, Kitas GD, Dimitroulas T, et al. Cardiovascular magnetic resonance in rheumatology: current status and recommendations for use. Int J Cardiol. 2016;217:135-148.

17. Kitas GD, Gabriel SE. Cardiovascular disease in rheumatoid arthritis: state of the art and future perspectives. Ann Rheum Dis. 2011;70:8-14.

18. Maradit-Kremers H, Crowson CS, Nicola PJ, et al. Increased unrecognized coronary heart disease and sudden deaths in rheumatoid arthritis: a population-based cohort study. Arthritis Rheum. 2005;52(2):402-411.

19. Stamatelopoulos KS, Kitas GD, Papamichael CM, et al. Atherosclerosis in rheumatoid arthritis versus diabetes: a comparative study. Arterioscler Thromb Vasc Biol. 2009;29(10):1702-1708.

20. Mavrogeni S, Karabela G, Stavropoulos E, et al. Imaging patterns of heart failure in rheumatoid arthritis evaluated by cardiovascular magnetic resonance. Int J Cardiol. 2013;168(4):4333-4335.

21. Rozin AP, Hasin T, Toledano K, Guralnik L, Balbir-Gurman A. Seronegative polyarthritis as severe systemic disease. Neth $J$ Med. 2010;68(6):236-241.

22. Tincani A, Rebaioli CB, Taglietti M, ShoenfeldY. Heart involvement in systemic lupus erythematosus, anti-phospholipid syndrome and neonatal lupus. Rheumatology. 2006;45 (Suppl 4):iv8-13.

23. Ishimori ML, Martin R, Berman DS, et al. Myocardial ischemia in the absence of obstructive coronary artery disease in systemic lupus erythematosus. JACC Cardiovasc Imaging. 2011;4:27-33.

24. O'Neill SG, Woldman S, Bailliard F, et al. Cardiovascular magnetic resonance imaging imaging in patients with systemic lupus erythematosus. Ann Rheum Dis. 2009;68:1478-1481.

25. Jennette JC, Falk RJ, Andrassy K, et al. Nomenclature of systemic vasculitides. Proposal of an international consensus conference. Arthritis Rheum. 1994;37:187-192.

26. Miloslavsky E, Unizony S. The heart in vasculitis. Rheum Dis Clin North Am. 2014;40(1):11-26.

27. Sunderkötter C, Sindrilaru A. Clinical classification of vasculitis. Eur J Dermatol. 2006;16:114-124.

28. Gonzales Lopez L, Gamez-Nava JI, Sanchez L, et al. Cardiac manifestations in dermato-polymyositis. Clin Exp Rheumatol. 1996;14:373-379.

29. Steen V. The heart in systemic sclerosis. Curr Rheumatol Rep. 2004;6:137-140.

30. Ioannidis JP, Vlachoyiannopoulos PG, Haidich AB, et al. Mortality in systemic sclerosis: an international meta-analysis of individual patient data. Am J Med. 2005;118:2-10.

31. Steen VD, MedsgerTA. Changes in causes of death in systemic sclerosis, 1972-2002. Ann Rheum Dis. 2007;66:940-944.

32. Tani C, Carli L, Vagnani S, et al. The diagnosis and classification of mixed connective tissue disease. J Autoimmun. 2014;48-49:46-49.
33. Greulich S, Deluigi CC, Gloekler S, et al. CMR imaging predicts death and other adverse events in suspected cardiac sarcoidosis. JACC Cardiovasc Imaging. 2013;6(4):501-511.

34. Mavrogeni S, Kouranos V, Sfikakis PP, et al. Myocardial stress perfusion-fibrosis imaging pattern in sarcoidosis, assessed by cardiovascular magnetic resonance imaging. Int J Cardiol. 2014;172(2): 501-503.

35. Douglas PS, Garcia MJ, Haines DE, et al. ACCF/ASE/AHA/ASNC/ HFSA/HRS/SCAI/SCCM/SCCT/SCMR 2011 Appropriate Use Criteria for Echocardiography. A Report of the American College of Cardiology Foundation Appropriate Use Criteria Task Force, American Society of Echocardiography, American Heart Association, American Society of Nuclear Cardiology, Heart Failure Society of America, Heart Rhythm Society, Society for Cardiovascular Angiography and Interventions, Society of Critical Care Medicine, Society of Cardiovascular Computed Tomography, Society for Cardiovascular Magnetic Resonance American College of Chest Physicians. American College of Cardiology Foundation Appropriate Use Criteria Task Force; American Society of Echocardiography; American Heart Association; American Society of Nuclear Cardiology; Heart Failure Society of America; Heart Rhythm Society; Society for Cardiovascular Angiography and Interventions; Society of Critical Care Medicine; Society of Cardio-vascular Computed Tomography; Society for Cardiovascular Magnetic Resonance; American College of Chest Physicians. J Am Soc Echocardiogr. 2011;24: 229-267.

36. Hachulla AL, Launay D, Gaxotte V, et al. Cardiovascular magnetic resonance imaging imaging in systemic sclerosis: a cross-sectional observational study of 52 patients. Ann Rheum Dis. 2009;68:1878-1884.

37. Raman SV, Aneja A, Jarjour WN. CMR in inflammatory vasculitis. J Cardiovasc Magn Reson. 2012;14:82.

38. Mavrogeni S, Spargias K, Markussis V, et al. Myocardial inflammation in autoimmune diseases: investigation by cardiovascular magnetic resonance and endomyocardial biopsy. Inflamm Allergy Drug Targets. 2009;8:390-397.

39. Greenwood JP, Maredia N, Younger JF, et al. Cardiovascular magnetic resonance and single-photon emission computed tomography for diagnosis of coronary heart disease (CE-MARC): a prospective trial. Lancet. 2012;379:453-460.

40. Brihaye B, Aouba A, Pagnoux C, Vignaux O, Le Hello C, Guillevin L. Rituximab reversed cardiac involvement of Wegener's granulomatosis: magnetic resonance imaging assessment. Presse Med. 2008;37: 412-415.

41. Allanore Y, Vignaux O, Arnaud L, et al. Effects of corticosteroids and immunosuppressors on idiopathic inflammatory myopathy related myocarditis evaluated by magnetic resonance imaging. Ann Rheum Dis. 2006;65:249-252.

42. Hunt SA, Abraham WT, Chin MH, et al; American College of Cardiology Foundation; American Heart Association. 2009 Focused update incorporated into the ACC/AHA 2005 Guidelines for the Diagnosis and Management of Heart Failure in Adults A Report of the American College of Cardiology Foundation /American Heart Association Task Force on Practice Guidelines Developed in Collaboration With the International Society for Heart and Lung Transplantation. $J$ Am Coll Cardiol. 2009;53(15):e1-e90.

43. Pieroni M, De Santis M, Zizzo G, et al. Recognizing and treating myocarditis in recent-onset systemic sclerosis heart disease: potential utility of immunosuppressive therapy in cardiac damage progression. Semin Arthritis Rheum. 2014;43(4):526-535.

44. Mavrogeni S, Bratis K, Sfendouraki E, Papadopoulou E, Kolovou G. Myopericarditis, as the first sign of rheumatoid arthritis relapse, evaluated by Cardiovascular magnetic resonance imaging. Inflamm Allergy Drug Targets. 2013;12(3):206-211. 
The Journal of Inflammation Research is an international, peer-reviewed open access journal that welcomes laboratory and clinical findings on the molecular basis, cell biology and pharmacology of inflammation including original research, reviews, symposium reports, hypothesis formation and commentaries on: acute/chronic inflammation; mediators of

Submit your manuscript here: https://www.dovepress.com/journal-of-inflammation-research-journa

inflammation; cellular processes; molecular mechanisms; pharmacology and novel anti-inflammatory drugs; clinical conditions involving inflammation. The manuscript management system is completely online and includes a very quick and fair peer-review system. Visit http://www.dove press.com/testimonials.php to read real quotes from published authors. 